\title{
Measurement of elastic properties of prostate cancer cells using AFM
}

\author{
Elsa Correia Faria, ${ }^{*} \dagger^{a}$ Nan Ma, $+^{a}$ Ehsan Gazi, ${ }^{b}$ Peter Gardner, ${ }^{a}$ Mick Brown, ${ }^{b}$ Noel W. Clarke ${ }^{b}$ and \\ Richard D. Snook ${ }^{a}$
}

\author{
Received 27th February 2008, Accepted 24th June 2008 \\ First published as an Advance Article on the web 25th July 2008 \\ DOI: $10.1039 / b 803355 b$
}

\begin{abstract}
This communication reports that three prostate cancer cells of differing metastatic potential were discriminated based on their Young's moduli (LNCaP - 287 $\pm 52 \mathrm{~N} \mathrm{~m}^{-2}, \mathrm{PC}-3-$ $1401 \pm 162 \mathrm{~N} \mathrm{~m}^{-2}$ and $\mathrm{BPH}-2797 \pm 491 \mathrm{~N} \mathrm{~m}^{-2}$ ) which were determined using AFM and the Hertz model.
\end{abstract}

\section{Introduction}

Prostate cancer is now the most commonly diagnosed cancer in men in western countries and there are 10000 deaths a year in the UK from this disease. ${ }^{1}$ The causes of prostate cancer are not yet known, but the largest risk factor is age; prostate cancer tends to affect men over the age of fifty with about half of all men having microscopic evidence of the disease at post-mortem by the age of 80. ${ }^{2}$ Currently, the American Cancer Society and the American Urologic Association recommend annual PSA (prostate specific antigen) screening and digital rectal examination (DRE) for all men above the age of 50 years with a life expectancy of ten years or greater. However, there are problems with this philosophy. In particular, the lack of sensitivity and specificity of PSA as a tool to diagnose prostate cancer, as well as PSA's inability to predict the clinical aggressiveness of the tumour, have limited its utility. ${ }^{3}$

From a therapeutic viewpoint, it is of crucial importance to evaluate a tumour's aggressive potential, as $c a$. $86 \%$ of men diagnosed with prostate cancer do not die from the disease, ${ }^{4}$ and those who do, die from metastatic spread. Currently, the clinical aggressiveness of a prostate tumour is determined by evaluating the microscopic tissue morphology (pathological grading) of biopsy tissue using the Gleason scoring system. However, this method is subjective and there is significant intra- and interobserver variation. ${ }^{5}$

Several studies have shown that cancer cells are more easily deformed than healthy cells and that highly aggressive cancer cells are more easily deformed than less aggressive cancer cells, ${ }^{6-12}$ and it has been suggested that the elastic properties of cancer cells play a major role in the metastatic process. ${ }^{9}$ This can be understood in light of the mechanism of metastasis in which cells invade neighbouring tissue. To do this they penetrate the basal lamina of the endothelium and the endothelial cells

${ }^{a}$ School of Chemical Engineering and Analytical Science, Manchester Interdisciplinary Biocentre, The University of Manchester, 131 Princess Street, Manchester, UKM1 7DN.E-mail: elsa.faria@manchester.ac.uk ${ }^{b}$ Genito-Urinary Cancer Research Group, Paterson Institute for Cancer Research, The University of Manchester, Christie Hospital NHS Trust, Manchester, UK M20 4BX

$\dagger$ Elsa Correia Faria and Nan Ma are joint first authors.

$\$$ Nan Ma is now at SUPA, School of Physics and Astronomy, University of St. Andrews, North Haugh, St. Andrews, Fife, UK KY16 9SS. themselves, to intravasate into the blood stream, where they have to survive the blood flow, and then extravasate into the tissue at the secondary site, where they will form a metastasis. ${ }^{13}$ Consequently, it has been hypothesised that elastic properties such a deformability of the cells could be used as a marker for metastatic potential. ${ }^{11,12,14}$ Since the pioneering work of Tao et $a{ }^{15}$ AFM has been widely used to determine the elastic properties of biological materials such as cells and has most recently been used by Cross et al. to study cells from patients suspected to have cancer ${ }^{14}$ ex vivo. They found that benign cells were $70 \%$ stiffer than cancer cells, but the correlation between metastatic potential and elasticity was not studied.

In the present paper we extend this approach to determine whether prostate cells of different stages in the disease progression can be discriminated based on their elastic properties, i.e. whether we can not only discriminate between non-cancerous and cancerous, but also between cancerous, non-metastatic and metastatic cells. The elastic moduli of whole cells were thus obtained for three prostate cell lines by indentation with an AFM tip whilst measuring the force applied. The elastic modulus was then obtained from these force curves using the Hertz model. The cell lines investigated were, primary BPH (benign prostate hyperplasia), a benign condition commonly found in older men, ${ }^{16}$ and two well-characterised and widely used prostate cancer cell lines LNCaP clone FGC and PC-3. ${ }^{17}$ These cells were selected on the basis of their benign (BPH) and malignant (PC3 and $\mathrm{LNCaP}$ ) characteristics and metastatic tendencies. $\mathrm{BPH}$ cells were isolated from primary tissue obtained with informed consent from males undergoing transurethral resection to treat for urinary outflow obstructive symptoms caused by BPH. A fourth cell line, PNT2-C2 was also tested for comparison, to ensure that the observed differences were not due to cells being grown using different culture media.

\section{Experimental}

\section{Cell culture}

LNCaP clone FGC and PNT2-C2 cells were cultured in RPMI 1640 (Cambrex UK, Wokingham, UK) containing 1\% (2 mM) L-glutamine (Sigma UK, Poole, UK) and 10\% FCS (Foetal Calf Serum, Invitrogen UK Ltd., Paisley, UK). PC-3 cells were cultured in Hams F-12 (Cambrex UK, Wokingham, UK) containing 7\% FCS and 1\% (2 mM) L-glutamine. BPH cells were isolated by collagenase digestion followed by differential centrifugation as described by Lang S.H. et al. ${ }^{18}$ They were then cultured in keratinocyte-serum-free media containing 1\% L-glutamine, 0.004\% EGF (Epidermal Growth Factor) and 5\% 
BPE (Bovine Pituitary Extract) (Invitrogen UK Ltd., Paisley, UK). All cells were grown at $37^{\circ} \mathrm{C}$ in a humidified atmosphere of $5 \% \mathrm{CO}_{2}$ in air until $70 \%$ confluent. Samples of cells at 3 to 4 different passages were tested for each cell line.

Cells to be analysed using the AFM were grown in their respective growth medium directly onto microscope slides placed in 12-well plates $\left(\mathrm{ca} .7 \times 7 \mathrm{~mm}^{2}\right.$ sterilised for at least $30 \mathrm{~min}$ in 70 $80 \%$ ethanol) until $70-80 \%$ confluent. The cells were adherent cells and therefore, no fixation or drying step was involved during sample preparation.

\section{AFM measurements}

Force curves were obtained using a commercially available Picoforce Multimode AFM (Veeco, Cambridge, UK) using vshaped silicon nitride cantilevers (Veeco, Cambridge, UK) of $196 \mu \mathrm{m}$ nominal length and nominal stiffness of $0.06 \mathrm{~N} \mathrm{~m}^{-1}$. The cantilever stiffness was calibrated using the inbuilt Thermal Tune method.

For each cell line, 3 to 4 samples of cells grown on different days (i.e. of different passages) were tested, except for BPH which were all prepared freshly and used from passage one. 15 to 22 individual cells of each cell sample were selected to obtain force curves and experiments were carried out in culture medium at room temperature. The ramp size was $20.55 \mu \mathrm{m}$ and the velocity of the AFM tip was $5.70 \mu \mathrm{m} \mathrm{s}^{-1}$ for all the measurements. To ensure that the cells were viable and under physiological conditions whilst the experiments were carried out, the indentation curves of the cells were acquired with the cells in their respective cell culture medium at room temperature.

To avoid influence from neighbouring cells, cells which were separate from other cells were selected to obtain force curves. The cells were mostly spherical and thus to minimise the effect of the substrate on the measurement the indentation was carried out in the centre of the cell. Although the size of the cells did not vary much ( $c a .20 \mu \mathrm{m}$ in diameter), similar sized cells were selected for the measurement in order to eliminate any potential differences in elastic properties caused by differences in size. The apparent elastic moduli were determined by fitting the Hertz model derived for a pyramid punch, ${ }^{19}$ to the experimental data using the Levenberg-Marquardt algorithm for non-linear least squares.

\section{Results and discussion}

Force-indentation curves were obtained for $52 \mathrm{LNCaP}, 53 \mathrm{PC}-3$ and $47 \mathrm{BPH}$ cells to which the Hertz model was fitted to the contact region up to an indentation of $3000 \mathrm{~nm}$. It was found that the Hertz model fits well to the curves in that indentation range, as exemplified in Fig. 1 for a BPH cell. The average apparent Young's moduli obtained for the three cell lines are shown in Fig. 2; these are within the range reported in the literature for other types of cells ${ }^{14,20}$ and show that the Young's moduli obtained for PC-3 cells were significantly different from those obtained for BPH and LNCaP cells, thus allowing the discrimination of different cell types.

A paired Student's $t$-test showed that the Young's modulus of the PC-3 cells is significantly different to that of the BPH cells $(t=3.09$, degrees of freedom $=98)$ and from that of the LNCaP

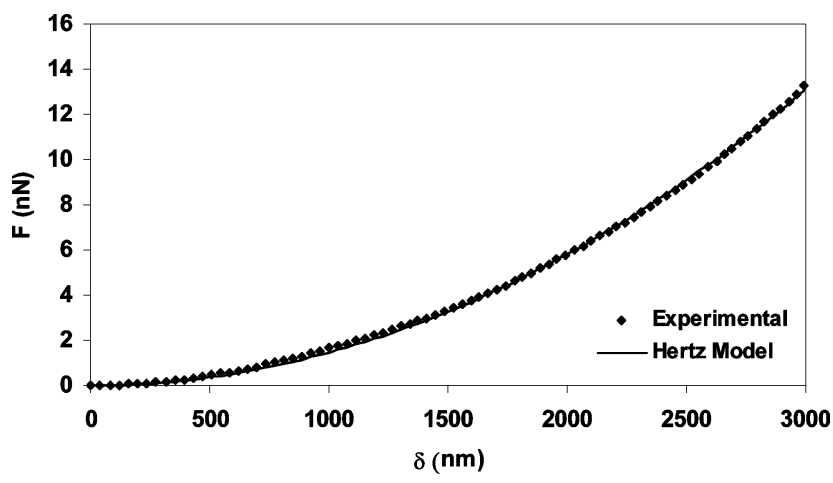

Fig. 1 Example of Hertz model fitting to a force curve obtained on a BPH cell.

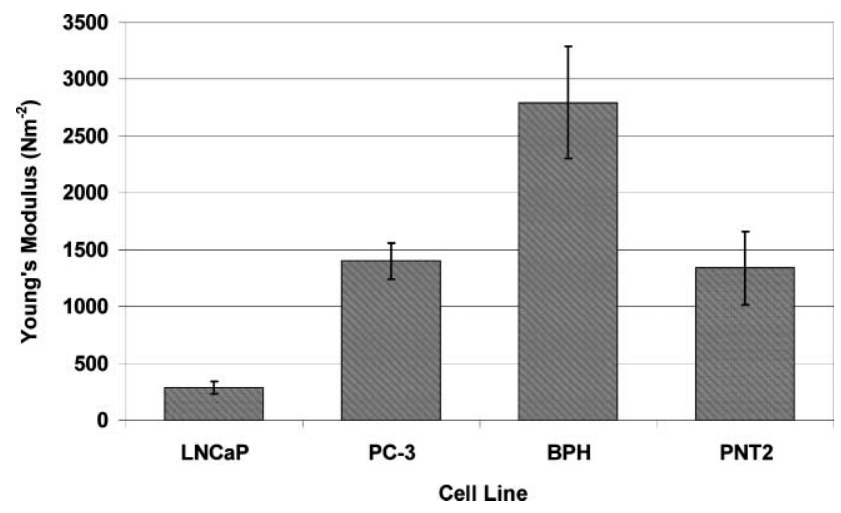

Fig. 2 Apparent Young's moduli for $\operatorname{LNCaP}\left(E=287 \pm 52 \mathrm{~N} \mathrm{~m}^{-2}, n=\right.$ 52), PC-3 ( $\left.E=1401 \pm 162 \mathrm{~N} \mathrm{~m}^{-2}, n=53\right) \mathrm{BPH}\left(E=2797 \pm 491 \mathrm{~N} \mathrm{~m}^{-2}\right.$, $n=47)$ and PNT2-C2 $\left(E=1139 \pm 320 \mathrm{~N} \mathrm{~m}^{-2}, n=48\right)$ cells. Error bars represent the $95 \%$ confidence interval.

cells $(t=5.67$, degrees of freedom $=103)$ at the $5 \%$ level and consequently that the Young's moduli of $\mathrm{LNCaP}$ and $\mathrm{BPH}$ were also significantly different at the same level $(t=6.12$, degrees of freedom $=97$ ).

Because the cells from the different cell lines were all grown in different media it was necessary to establish whether the observed differences were due to the different growth conditions or due to differences in cell physiology. To this end, cells from the established PNT2-C2 cell line, which was established by immortalisation of normal prostatic epithelial cells ${ }^{21}$ were tested in the same way as the other cells. These cells were chosen as they were grown in the same culture medium as LNCaP clone FGC cells. The average Young's modulus for this cell line was $1139 \pm 320 \mathrm{~N} \mathrm{~m}^{-2}(n=48)$ and differed significantly from that obtained for LNCaP cells $(t=5.33$, degrees of freedom $=98)$ at the $5 \%$ level. The modulus obtained for the PNT2-C2 cells however was not significantly different at the $5 \%$ level from that of the PC-3 cells $(t=0.227$, degrees of freedom $=51)$, which were grown in different medium. The results obtained are shown in Fig. 2. This result shows that the PNT2-C2 cells and the LNCaP cells which were grown in the same medium, had significantly different Young's moduli and that the PNT2-C2 cells and the PC-3 cells which were grown in different medium had moduli which were not significantly different; this indicates that the differences in Young's modulus observed for the other three cell lines were not due to the cells being grown in different medium 
and that the cells can indeed be discriminated based on their Young's modulus.

As hypothesised, the benign BPH cells are less easily indented and therefore exhibit a higher Young's modulus than the tumorigenic LNCaP and PC-3 cells. However, based on the hypothesis it was expected that the highly invasive PC-3 cells would have a lower Young's modulus than the noninvasive LNCaP cells. This was not the case. Similarly, Zhang et $a .^{22}$ observed that the elastic coefficients for hepatocellular carcinoma cells were significantly higher than that of human hepatocytes, and the work of Darling et $a .^{23}$ did not show a correlation between malignancy and elastic modulus or other biomechanical properties of chondrosarcoma cells. The reasons for this behaviour are not yet know, but are currently being investigated by the authors.

In summary, it has been shown that AFM is a useful tool in the study of the mechanical properties of cells and in particular to determine relative Young's moduli of prostate cells of different metastatic potential. Previous work has demonstrated that the cells used in this study could be discriminated using infrared microscopy and photoacoustic spectroscopy, which both provide information on the molecular differences of the cells. ${ }^{24,25}$ Together, the elasticity and spectroscopic information might be used to distinguish prostate cancer cells of varying aggressiveness and possibly enable us to gain further information to help elucidate the mechanisms of metastasis and plan new forms of diagnosis and therapy. Together these techniques might also prove to be invaluable in the study of the effect of potential and established cancer drugs on anti-cancer cells.

\section{Conclusions}

In this study the Young's moduli of three different prostate cell lines were determined by indentation with a pyramidal AFM tip and applying the Hertz model to the force curves obtained. It was found that the Hertz model fitted the experimental data very well in the indentation range $0-3000 \mathrm{~nm}$ and that the Young's moduli of the different cell lines were significantly different from each other. Thus it was possible to discriminate between the three cell lines even with relatively few cells tested (52 LNCaP, 53 PC-3 and 47 BPH cells). Furthermore, as was expected the Young's modulus determined for $\mathrm{BPH}$ was higher than that of the cancer cells. However, the highly invasive PC-3 cells were found to have a higher Young's modulus than the non-invasive LNCaP clone FGC cells, showing that there are variations between different types of prostate cancer cells, a feature which is reflected in their clinical behaviour

Much work has yet to be carried out to elucidate the relationship between metastatic potential and the mechanical properties of cells. However, these results distinctly show that when tested under the same controlled experimental conditions the three cell lines tested have significantly different Young's moduli allowing discrimination using AFM indentation and the Hertz model.

\section{Acknowledgements}

The authors would like to acknowledge AICR for funding (E. Gazi).

\section{References}

1 Cancer Research UK Website, 2007, http://info.cancerresearchuk. org/cancerstats/types/prostate/ $? \mathrm{a}=5441$.

2 G. P. Haas and W. A. Sakr, Ca Cancer J. Clin., 1997, 47, 273-287.

3 J. Concato, C. K. Wells, R. I. Horwitz, D. Penson, G. Fincke, D. R. Berlowitz, G. Froehlich, D. Blake, M. A. Vickers, G. A. Gehr, N. H. Raheb, G. Sullivan and P. Peduzzi, Arch. Intern. Med., 2006, 166, 38-43.

4 L. Klotz, Curr. Treat. Options Oncol., 2006, 7, 355-362.

5 J. B. Lattouf and F. Saad, BJU Int., 2002, 90, 694-698.

6 K. F. Tullberg and M. M. Burger, Invasion Metastasis, 1985, 5, 1-15.

7 B. Lincoln, H. M. Erickson, S. Schinkinger, F. Wottawah, D. Mitchell, S. Ulvick, C. Bilby and J. Guck, Cytometry, Part A, 2004, 59, 203-209.

8 M. Lekka, P. Laidler, D. Gil, J. Lekki, Z. Stachura and A. Z. Hrynkiewicz, Eur. Biophys. J., 1999, 28, 312-316.

9 K. A. Ward, W. I. Li, S. Zimmer and T. Davis, Biorheology, 1991, 28, 301-313.

10 T. Ochalek, F. J. Nordt, K. Tullberg and M. M. Burger, Cancer Res., $1988,48,5124-5128$.

11 O. Thoumine and A. Ott, Biorheology, 1997, 34, 309-326.

12 J. Guck, S. Schinkinger, B. Lincoln, F. Wottawah, S. Ebert, M. Romeyke, D. Lenz, H. M. Erickson, R. Ananthakrishnan, D. Mitchell, J. Kas, S. Ulvick and C. Bilby, Biophys. J., 2005, 88, 36893698.

13 R. G. Vile, in Cancer Metastasis: From Mechanism to Therapies, ed. R. G. Vile, John Wiley \& Sons, Chichester, 1995, pp. 21-46.

14 S. E. Cross, Y.-S. Jin, J. Rao and J. K. Gimzewski, Nat. Nanotechnol., 2007, 2, 780-783.

15 N. J. Tao, S. M. Lindsay and S. Lees, Biophys. J., 1992, 63, 1165-1169.

16 J. E. McNeal, Am. J. Surg. Pathol., 1988, 12, 619-633.

17 R. E. Sobel and M. D. Sadar, J. Urol., 2005, 173, 342-359.

18 S. H. Lang, N. W. Clarke, N. J. R. George, T. D. Allen and N. G. Testa, Prostate, 1998, 34, 203-213.

19 G. G. Bilodeau, J. Appl. Mech., Trans ASME, 1992, 59, 519-523.

20 M. Radmacher, IEEE Eng. Med. Biol. Mag., 1997, 16, 47-57.

21 The European Collection of Cell Cultures, 2007, http://www. ecacc.org.uk.

22 G. Zhang, M. Long, Z. Z. Wu and W. Q. Yu, World J. Gastroenterol., 2002, 8, 243-246.

23 E. M. Darling, S. Zauscher, J. A. Block and F. Guilak, Biophys. J., 2007, 92, 1784-1791.

24 T. J. Harvey, A. Henderson, E. Gazi, N. W. Clarke, M. Brown, E. C. Faria, R. D. Snook and P. Gardner, Analyst, 2007, 132, 292-295.

25 E. Gazi, J. Dwyer, P. Gardner, A. Ghanbari-Siahkali, A. P. Wade, J. Miyan, N. P. Lockyer, J. C. Vickerman, N. W. Clarke, J. H. Shanks, L. J. Scott, C. A. Hart and M. Brown, J. Pathol., 2003, 201, 99-108. 